\title{
Study on Detection of P300 ERP Component in EEG Signals and Algorithms
}

\author{
Syed Kamran Haider, Ai-Min JIANG, Shahzad Ashraf,Inam Ullah \\ Internet of Things (IOT), Hohai University \\ Changzhou, China \\ e-mail: kamranhaider85@yahoo.com, jiangam@hhu.edu.cn, engr_shahzadarain@yahoo.com, \\ engr.inamdir58@yahoo.com
}

\begin{abstract}
Keywords: component; Lie detection, P300, extreme learning machine, event related potential. Abstract.P300 Event Related Potential (ERP) has drawn much interest in different application of psychological background. An endogenous ERP component, P300, has been widely inspected in detecting deception. Detecting P300 from EEG signals is hard to find because P300 is overloaded with noise and other EEG signals. We typically use the features of the amplitude and latency of P300 to recognize P300.In this paper we are presenting several methods for the efficient detection of P300 detection algorithms such as; reconstruction algorithm for small P300 wave using Independent Component Analysis (ICA), Extreme Learning Machine (ELM) method using BackPropagation network, Multi-domain EEG signal processing. Each detection method has good effectiveness but the complexation level is dissimilar for each algorithm based on the constraints and optimal features used at the classification stage. This survey will brings you algorithms for the P300 detection and give you detail idea about the use of them.
\end{abstract}

\section{Introduction}

Numerous studies have been conducted on lie detection in recent years, following with Event Related Potentials (ERP) [1]. The intimate ERP component i.e. P300 has been investigated in depth for the identification of deception. [2]. P300 element arouse from the random but, meaningful stimuli. Crest in P300 frequently depicts its occurrence at 300-1000 ms from the stimulus onset, and then this dormancy rises with intricacy of stimulus task. Recognizing P300 from EEG is not an easy job as P300 is superimposed actively on EEG, though normally amplitude \& latency features of P300 are used for the identification of P300.In response to this, determining innocent subjects \& guilty ones becomes harder since the signal to noise ratio (SNR) is quiet low when simple procedures are used for P300 extraction [3-4].

Various studies have been done on the detection of deception using neurophysiological signals such as Magnetic Resonance Imaging [5] and Event Related Potential (ERP) [6]. P300 (P3), an intimate ERP component, has been widely put into use used for lie detection [7]. On the contrary pattern recognition (PR) classifiers in lie detection have not been yet reported widely. Davatzikos et al. [8] proposed a support vector machine (SVM)-based method for the classification of arrangement of the activities of brain achieved during lying \& truth-telling by using fMRI data. Abootalebi et al. [9] used linear discrimination analysis (LDA) to identity P3 responses and obtained the most detection rate of $86 \%$. Gao et al. [10] used SVM for the first time for detection of lie, the outcome showed that SVM outperformed fisher discrimination analysis (FDA) and back-propagation neural network (BPNN) by obtaining the highest classification accuracy of $91.8 \%$.

A lot of real-word problems absorbs the simultaneous feature selection and tuning parameters for Support Vector Machines (SVM) as one of the advantageous method for classification of data. It is essential that selections of features \& parameters optimization needs to be carried out at the same time, since the kernel parameters are controlled by the feature subset choice $\&$ vice versa. Parameters like penalty parameter $\mathrm{C} \&$ kernel parameter $\gamma$ for radial basis function (RBF) kernel helps in achieving better results. 


\section{Methodology for P300 Detection}

\subsection{Reconstruction algorithm for small P300 wave using Independent Component Analysis (ICA)}

Presently, Number of researchers' averaged ERP data to enhance SNR relative to EEG activity and artifacts, that were non-phase \& non-time locked to the stimuli (e.g., Electrooculography, EOG). Since P300s shape of wave \& its amplitude varies significantly in each trial, the average method is unable to depict dynamic information relating to ERP. Recently, many researchers have investigated single trial-based lie detection procedures. Normally P300 features are extracted via single trial \& after that feature samples are used to train the classifier $[9,11]$ and then various brain states are further classified, \& then into deception \& finally in the end true tellers. Most recently, an independent component analysis (ICA) procedure [12-13] was used to extract the P300 [14], and outcomes depicts that the P300 from the decomposed components or reconstructed signals on electrodes were more categorized as compared to "sensor signals" (i.e., the SNR of P300 was higher as compared to initial sensor signals.) [15-16]. In this study, ICA method was adopted to enhance the SNR of P300 and hence improves the classification accuracy for lie detection.

1. De-noising procedure of $P 300$

The presented denoising and reconstruction procedure is divided into following three steps:

1.1. Let $\boldsymbol{I}_{\boldsymbol{c}}$ denote the waveform of the $c$ th IC. On the basis of mixing matrix $\mathbf{W}^{\mathbf{- 1}}$, denote the $i$ th column element by $W_{i j}^{-1}(i, j=1,2, \ldots \ldots, 12)$, and accordingly $W_{. j}^{-1}$ denoted all the elements in $j$ th column.

First, each matrix $\mathbf{W}^{-\mathbf{1}}$ was normalized to the other matrix $\mathbf{U}$ :

$$
U_{i j} \leftarrow\left(\left|W_{i j}^{-1}\right| / \max \left(\left|W_{i j}^{-1}\right|\right)\right)
$$

1.2. Let $U_{P_{z^{j}}}$ denote new projection strength of the $j$ th IC onto the Pz sensors. For the each matrix $\mathbf{U}$, Suppose that $\mathrm{S}$ is a vector $S=\left\{S_{j}, j=1,2, \ldots \ldots, 12\right\}$, and the value $S_{j}$ is calculated by $U_{i j}$ and given by:

$$
\begin{aligned}
& S_{j}=U_{P_{z^{j}}}+0.7 * U_{P_{3} j}+0.7 * U_{P_{4} j}+0.5 * \\
& U_{c_{z^{j}}}+0.3 * U_{O_{z^{j}}}+0.1 * U_{F_{z^{j}}}
\end{aligned}
$$

1.3. Sort $\left\{S_{j}, j=1,2, \ldots \ldots, 12\right\}$ in descending order. Let $\vec{S}$ denote the sorted vector with the sorted index vector represented by $\mathbf{V}$ in which the $j$ th element value denotes the position of the $\overrightarrow{S_{j}}$ value in unsorted vector $\mathrm{S}$.

1.4. Suppose $X_{P_{z} q}$ denote the reconstructed Pz waveform belonging to the $q$ th EEG dataset in the set $\mathbf{R} 1$ or R2, and then for each subject group, let $X=X_{P_{z^{1}}}, X_{P_{z^{2}}} \ldots X_{P_{z^{q}}}, \ldots X_{P_{z^{M}}}$ denote a vector set consisting of $\mathrm{M}$ different vector $X_{P_{z}}$. Finally, the reconstructed procedure can be given by:

$$
X_{p q}=\sum_{j=1}^{2} W_{P_{z} V_{j}}^{-1} \times I_{V_{J}}, q=1,2, \ldots \ldots, M
$$

Where the number of reconstructed components was set as 2. Let R-G denote the first vector set transformed from R1, and R-I denote the other from R2.

\subsection{Extreme Learning Machine (ELM)}

Extreme learning machine (ELM) was proposed by Huang et al. [18] to over throw some inherent drawbacks of SVM and BPNN. ELM randomly specifies the input weights and biases, and then analytically calculates the output weights with the smallest norm. Hence, ELM provides better generalization power at an efficiently faster training speed [19]. In recent decades huge attention has been achieved by ELM in various fields related to PR [20-21]. Till present, there isn't any appraisal that uses ELM for lie detection \& classification of guilty \& innocent subjects. To achieve a good 
harmony among high accuracy of lie detection \& short training time, ELM is acquainted to classify the 2 kinds of subjects (the guilty and the innocent) in this document. For ELM, though the number of hidden nodes (NHN) for ELM is haphazardly assigned in ordinary ELM algorithm, the classification boundary may not be the optimal one when this number remains unchanged during training procedure [22]. Furthermore, too many or few NHN might lead to over-fitting or underfitting problem [23]. It's the blazing topic going on connected to the analysis of ELM algorithm\& hence very few methods were proposed for its solution. [22, 24-26]. On one side, these enhanced ELM algorithms are quiet complex for online or real-time classification system. On the other hand assumption is made to have a closer relation among NHN \& dimension of feature space. Thus, in this study, NHN is not assigned for ELM, rather tuned it by using grid searching in order to decide its optimal values for classification. Furthermore, expectation was made that the training \& testing time could be significantly reduced as compared to SVM \& BPNN.

2. Algorithm

Given $\mathrm{N}$ different training samples $\left(x_{i}, t_{i}\right)$, where $x_{i}=\left[x_{i 1}, x_{i 2}, \ldots, x_{i n}\right]^{T} \epsilon R^{n}$ and $t_{i}=\left[t_{i 1}, t_{i 2}, \ldots, t_{i m}\right]^{T} \in R^{m}$, we train SLFN with $\mathrm{K}$ hidden nodes and activation function $g(x)$. this network can be mathematically modelled as:

$$
\begin{aligned}
& \sum_{i=1}^{k} \beta_{i} g_{i}\left(x_{j}\right)=\sum_{i=1}^{k} \beta_{i} g\left(a_{i} \times x_{j}+b_{i}\right)= \\
& t_{j}, j=1, \ldots, N
\end{aligned}
$$

Where $\boldsymbol{a}_{\boldsymbol{i}}=\left[a_{i 1}, a_{i 2}, \ldots, a_{i n}\right]^{T}$ denotes the weight vector connecting $i$ th hidden node and $n$ input nodes; $b_{i}$ is bias of $i$ th hidden node; $\boldsymbol{\beta}_{\boldsymbol{i}}=\left[\beta_{i 1}, \beta_{i 2}, \ldots, \beta_{i m}\right]^{T}$ denotes the weigh vector connecting $i$ th hidden node and the $m$ output nodes.

Above $\mathrm{N}$ equations can be rewritten in a matrix as:

$$
H \beta=T
$$

$$
\begin{aligned}
& \text { Where } \\
& \mathbf{H}= \\
& {\left[\begin{array}{ccc}
g\left(a_{1} \cdot x_{1}+b_{1}\right) & \cdots & g\left(a_{K} \cdot x_{1}+b_{K}\right) \\
\vdots & \cdots & \vdots \\
g\left(a_{1} \cdot x_{N}+b_{1}\right) & \cdots & g\left(a_{K} \cdot x_{N}+b_{K}\right)
\end{array}\right]_{N \times \boldsymbol{K}}} \\
& \boldsymbol{\beta}=\left[\begin{array}{c}
\boldsymbol{\beta}_{1}^{T} \\
\vdots \\
\boldsymbol{\beta}_{K}^{T}
\end{array}\right]_{K \times \boldsymbol{m}} \text { and } \boldsymbol{T}=\left[\begin{array}{c}
\boldsymbol{t}_{1}^{T} \\
\vdots \\
\boldsymbol{t}_{N}^{T}
\end{array}\right]_{N \times \boldsymbol{m}}
\end{aligned}
$$

$\mathbf{H}$ is called the hidden layer output matrix with the $i$ th column being the output of the $i$ th hidden node (Huang et al., 2000). To learn $N$ instances for SLFN one traditionally find the solution set $\mathbf{W}$, including $a_{i}, \beta_{i}$ and $b_{i}$, to minimize following cost function:

$$
E(\mathbf{W})=\sum_{j=1}^{N}\left(\sum_{i=1}^{K} \beta_{i} g\left(a_{i}-x_{j}+b_{i}\right)-t_{j}\right)^{2}
$$

Given an arbitrarily small value $\varepsilon>0$, Huang et al. [18] proved that if the input weights and biases of hidden nodes are assigned randomly and the activation function in the SLFN is infinitely differentiable, the SLFN can approximate the $N$ training data with $\varepsilon$ error, i.e., $\|\boldsymbol{H} \beta-\boldsymbol{T}\| \leq \varepsilon$. In this case, matrix $\mathbf{H}$ has been randomly fixed. Hence, the training procedure of SLFN is equal to seeking a least- squares (LS) solution of the linear system.

\subsection{Multi-domain EEG signal processing Method}

The architecture of the proposed method consists of multi-domain preprocessing module, multidomain feature extraction module, feature selection module based on genetic algorithm (GA) [27] and classification module based on linear discriminant analysis (LDA) [28]. 


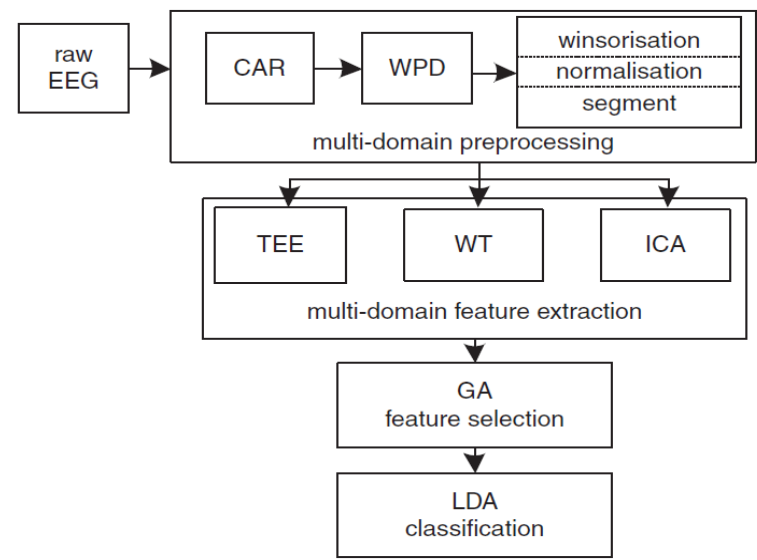

Fig.1 Architecture of multi-domain EEG signal processing method used for P300-based deception detection in simulated network fraud condition

The multi-domain preprocessing module performed common average reference (CAR) [29] as spatial filter, wavelet packet decomposition (WPD) [27] as temporal-spectral filter, winsorisation [30], normalization and segment as temporal filter. In the feature extraction module, multi-domain features were being extracted from temporal, temporal-spectral \& spatial domains using temporal energy entropy (TEE), wavelet transform (WT) [28] and independent component analysis (ICA), respectively [28].

After the CAR, the EEG signals were band-pass filtered from 0.25 to $30 \mathrm{~Hz}$ by WPD with Daubechies 4 function used as mother wavelet. In the temporal preprocessing phase, winsorisation procedure [30] was being applied to replace the largest \& smallest 5\% amplitude of EEG signals with corresponding threshold. After this, EEG signals were normalized into $[-1,+1]$ before they can be divided into epochs with 1000 ms length after stimulus onset. Furthermore, temporal domain analysis was implemented after the above preprocessing by averaging the EEG signals of all epochs across 12 subjects in $\mathrm{Fz}, \mathrm{Cz}$ and $\mathrm{Pz}$ electrodes. The results depicted that, in the time interval between 200 and $600 \mathrm{~ms}$ after the stimulus onset, a larger P300 component was evoked by P stimulus as predicted. Hence, only the signals in this time interval were considered in the following procedure. During the multi-domain feature extraction process, TEEs were extracted as temporal features. The TEE for EEG signal is defined as:

$$
E_{i}=-\log _{2}\left(P_{i} / \sum_{i=1}^{n} P_{i}\right)
$$

where $P_{i}$ and $E_{i}$ are the energy and TEE of the $i$ th segment of a single epoch EEG, and the single epoch EEG is divided into $n$ segments (i.e., $n=10$ ). The TEEs in Fz, $\mathrm{Cz}$ and $\mathrm{Pz}$ were average to obtain ten temporal features named TEE1-TEE10. 


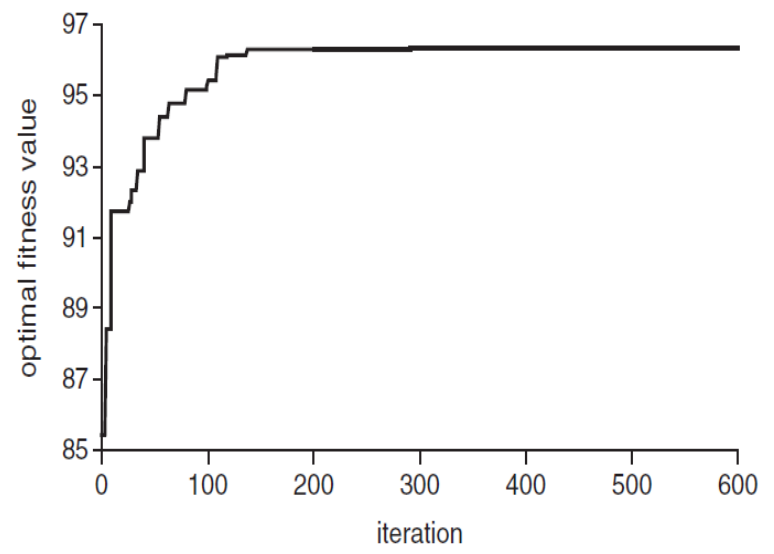

Fig.2 Optimal fitness value in each generation during GA procedure

While performing the calculation of temporal-spectral features \& spatial features, WT \& ICA were performed. After that power information of wavelet approximate coefficients in different electrodes was averaged in order to obtain 19 temporal-spectral features called WT1-WT19, and the 9 elements of mixing matrix in ICA, named $w^{\prime}{ }_{11}-w^{\prime} 33$, were regarded as spatial features to denote the spatial distribution characteristic of the EEG. Finally, the above features were combined to obtain a multi-domain feature set, and GA was implemented to search an optimal feature subset (OFSS) with the classification accuracy of the LDA classifier used as fitness value during the GA procedure.

\section{Discussion}

In this paper, small-number of stimuli-based detection method is proposed, in which using ICA to improve the SNR of P300, is the main difference among proposed method \& conventional machine learning methods. Firstly, the SNR of P300 in a single trial, we assume, is quiet low. Hence, problem related to all ERP based lie detection methods. In order to decrease the effect of noise (e.g., EOG spontaneous EEG) on the performance of detection to a greater extent, ICA procedure was used to separate out P300 from other noise sources.

ELM possess some fine features as compared to other machine learning algorithms. For instance, there is almost no classifier parameter required to be tuned in its learning procedure compared to BPNN \& SVM that significantly reduces the computational cost and training complexity. Secondly, the learning speed is quiet slow when a large number of training data need to learn and hence too slow to meet the requirements of real-time applications. Last but not the least artificial neural networks such as BPNN are prone to fall into a local minimum. Contrary to this, ELM has a surprisingly greater learning speed as the output weights are determined analytically rather than by using iterative strategy (Huang et aI., 2004, 2006a).

P300-based deception detection uses 3-stimulus. GKT technique in simulated network fraud condition, \& proposed a multi-domain EEG signal processing method used in deception detection experiments.

\section{Acknowledgment}

This work was supported in part by the National Nature Science Foundation of China under grants 61101158, 61471157, and 61401148, and the Natural Science Foundation of Jiangsu Province, China under grants BK20130238, BK20141159 and BK20141157, and Science \&Technology Program of Changzhou, China under Grant CJ20159039. 


\section{References}

[1]J. P. Rosenfeld, "Event-related potentials in the detection of deception,". Handbook of Polygraph Testing. Academic Press, NewYork, pp. 265-286., 2002.

[2]E. H. Meijer, F. T. Y. Smulders, H. L. G. J. Merckelbach, A. G. Wolf, "The P300 is sensitive to concealed face recognition. International," Journal of Psychophysiology, vol 66, pp. 231-237, 2007.

[3]V. Abootalebi, M. H. Moradi, M. A. Khalilzadeh, "A new approach for EEG feature extraction in P300-based lie detection," Computer Methods and Programs in Biomedicine, vol 94, pp. 48-57. 2009.

[4]J. P. Rosenfeld, M. Soskins, G.Bosh, A. Ryan, "Simple, effective countermeasures to P300based tests of detection of concealed information," Psychophysiology, vol 41, 205-219, 2004.

[5] Langleben, D.D., Loughead, 1. W., Bilker, W.B., et ai. "Telling truth from lie in individual subjects with fast event-related fMRl", Human Brain Mapping, 2005, 26, pp. 262-272.

[6]Rosenfeld, 1P., "Event-related potentials in the detection of deception", Handbook of Polygraph Testing, Academic Press, New York, 2002, pp. 265-286.

[7]Meijer, E.H., Smulders, F.T.Y., Merckelbach, H.L.G. Wolf., 1A.G., "The P300 is sensitive to concealed face recognition", International Journal of Psychophysiology, 2007, 66, pp. 231-237.

[8]Davatzikos, c., Ruparel, K., Fan, Y., et al. "Classitying spatial patterns of brain activity with machine learning methods: Application to lie detection", Neurolmage, 2005, 28, pp. 663-668.

[9]Abootalebi, V., Moradi, M.H., Khalilzadeh, M.A., "A new approach for EEG feature extraction in P300-based lie detection", Computer Methods and Programs in Biomedicine, 2009, 94, pp. 48-57.

[10] Gao, IF., Van, X.G., Sun, IC., Zheng, c.X., "Denoised P300 and machine learning-based concealed information test method", Computer Methods and Programs in Biomedicine, 2011, 104, pp. 410-417.

[11] J. P. Rosenfeld, M. Soskins, G.Bosh, A. Ryan, "Simple, effective countermeasures to P300based tests of detection of concealed information," Psychophysiology, vol 41, 205-219, 2004.

[12] C. Davatzikos, K. Ruparel, Y. Fan, D. G. Shen, M. Acharyya, J. W. Loughead, R. C. Gur, D. D. Langleben, "Classifying spatial patterns of brain activity with machine learning methods: Application to lie detection," NeuroImage, vol 28, pp. 663-668, 2005.

[13] T. P. Jung, S. Makeig, C. Humphries, T. W. Lee, M. J. McKeown, V. Iragui, T. J. Sejnowski, "Removing electroencephalographic artifacts by blind source separation," Psychophysiology, vol 37, pp. 163-178, 2000.

[14] L. Parra, P. Sajda, "Blind source separation via generalized eigenvalue decomposition," J. Machine Learning Res. , vol 4, pp. 1261-1269, 2003.

[15] C. T. Lin, I. F. Chung, L. W. Ko, Y. C. Chen, S. F. Liang, J. R. Duann, "EEG-Based Assessment of Driver Cognitive Responses in a Dynamic Virtual-Reality Driving Environment," IEEE Transaction on Biomedical Engineering, vol 54, pp. 1394-1352, 2007.

[16] C. I. Hung, P. L. Lee, Y. T. Wu, L. F. Chen, T. CH. Yeh, "Recognition of Motor Imagery Electroencephalography Using Independent Component Analysis and Machine Classifiers," Annals of Biomedical Engineering, vol 33, pp. 1053-1070, 2005.

[17] A. C. Tang, M. T. Sutherland, Y. Wang, "Contrasting single-trial ERPs between experimental manipulations: Improving differentiability by blind source separation," NeuroImage, vol 29 pp. 335-346, 2006. 
[18] Huang, G.B., Zhu, Q.Y., Siew, C.K., "Extreme learning machine: theory and applications", Neuro computing, 2006, 70, pp. 489-501.

[19] Huang, G.B., Wang, D.H., Lan, Y., "Extreme learning machines: a survey", International Journal of Machine Learning and Cyber, 2011, 2, pp. 107-122.

[20] Deng, W., Chen, L., "Color image watermarking using regularized extreme learning machine", Neural Network World, 2010, 20, pp. 317-330.

[21] Chen, F.L., Ou, T.Y, "Sales forecasting system based on gray extreme learning machine with Taguchi method in retail industry", Expert System Application, 2011, 38, pp. 1336-1345.

[22] Cao, J.W., Lin, Z.P., Huang, G.B., " Composite Function Wavelet Neural Networks with Differential Evolution and Extreme Learning Machine ", Neural Processing Letters, 2011, 33, pp. 251-265.

[23] Shi, L.C., Lu, B.L, "EEG-based vigilance estimation using extreme learning machines", Neurocomputing, http://dx.doi.orgll 0.10 16/j.neucom, 2012.

[24] Huang, G.B., Chen, L. "Enhanced random search based incremental extreme learning machine",Neurocomputing, 2008, 71, pp. 3460-3468.

[25] Miche, Y., Bas, P., Jutten, C., et aI., "A methodology for building regression models using extreme learning machine: Op-elm", Proceedings of the 16th European Symposium on Artificial Neural Networks, Bruges, Belgium, 2008, pp. 1-6.

[26] Yuan, L., Yeng, C.S., Huang, G.B., "A constructive enhancement for Online Sequential Extreme Learning Machine", Neural Networks, International Joint Conference, 2009, 1708-1713.

[27] Ocak, H.: 'Optimal classification of epileptic seizures in EEG using wavelet analysis and genetic algorithm', Signal Process., 2008, 88, (7), pp. 1858-1867

[28] Bashashati, A., Fatourechi, M., Ward, R.K., and Birch, G.E.: 'A survey of signal processing algorithms in brain-computer interfaces based on electrical brain signals', J. Neural Eng., 2007, 4, (2), p. R32

[29] Ludwig, K.A., Miriani, R.M., Langhals, N.B., Joseph, M.D., Anderson, D.J., and Kipke, D.R.: 'Using a common average reference to improve cortical neuron recordings from microelectrode arrays', J. Neurophysiol., 2009, 101, (3), pp. 1679-1689

[30] Savage, G.R., Saling, M.M., Davis, C.W., and Berkovic, S.F.: 'Direct and indirect measures of verbal relational memory following anterior temporal lobectomy', Neuropsychologia, 2002, 40, (3), pp. 302-316. 